\title{
IFN- $\gamma$ protects short-term ovarian carcinoma cell lines from CTL lysis via a CD94/NKG2A-dependent mechanism
}

\author{
Karl-Johan Malmberg, ${ }^{1}$ Victor Levitsky, ${ }^{2}$ Håkan Norell, ${ }^{1}$ Cristina Teixeira de Matos, ${ }^{2}$ \\ Mattias Carlsten, ${ }^{1}$ Kjell Schedvins, ${ }^{3}$ Hodjattallah Rabbani, ${ }^{1}$ Alessandro Moretta, ${ }^{4}$ \\ Kalle Söderström, ${ }^{2}$ Jelena Levitskaya, ${ }^{1}$ and Rolf Kiessling ${ }^{1}$
}

\begin{abstract}
${ }^{1}$ Immune and Gene Therapy Laboratory, Cancer Center Karolinska, Department of Oncology and Pathology, ${ }^{2}$ Microbiology and Tumor Biology Center, Karolinska Institutet, Stockholm, Sweden ${ }^{3}$ Department of Obstetrics and Gynecology, Karolinska Hospital, Stockholm, Sweden ${ }^{4}$ Dipartimento di Medicina Sperimentale, Università di Genova, Genova, Italy
\end{abstract}

IFN- $\gamma$ regulates the immunogenicity of target cells by increasing their expression of HLA class I molecules. This facilitates the $\mathrm{T}$ cell receptor-mediated recognition by $\mathrm{CD}^{+} \mathrm{T}$ cells but decreases target cell sensitivity to lysis by NK cells due to engagement of inhibitory NK receptors. In this study, short-term tumor cell lines from patients with advanced ovarian carcinomas were established. We demonstrate the paradoxical finding that IFN- $\gamma$ treatment of these short-term ovarian carcinoma cell lines (OVACs) resulted in resistance of tumor cells to lysis by peptide- and allospecific $\mathrm{CD8}^{+} \mathrm{T}$ cells. Blocking experiments revealed that this phenomenon was dependent on enhanced inhibitory signalling via CD94/NKG2A receptors expressed on the effector cells. This was associated with increased expression of HLA-E mRNA and HLA-G at the protein level in IFN- $\gamma$-treated OVACs. Furthermore, pulsing of untreated OVACs with the leader sequence peptide of HLA-G protected these cells from lysis by CTLs, thus mimicking the inhibitory effect of IFN- $\gamma$. This study provides evidence that $\mathrm{CD} 94 / \mathrm{NKG} 2 \mathrm{~A}$ receptors play an important role in regulating $\mathrm{T}$ cell activity against tumors and shows that IFN- $\gamma$ modulation of target cells may shift the balance of triggering and inhibitory signals to $T$ cells, turning off their cytolytic activity.

J. Clin. Invest. 110:1515-1523 (2002). doi:10.1172/JCI200215564.

\section{Introduction}

IFN- $\gamma$ is an important regulator of the immune response to tumors. The significance of IFN- $\gamma$ in relation to tumor immunity has recently been demonstrated in a model of IFN- $\gamma$ receptor knockout mice (1). Mice that are deficient in IFN- $\gamma$ signalling develop spontaneous tumors at a higher frequency than do normal mice. Furthermore, IFN- $\gamma$ prevents carcinogen-induced tumor formation in mice $(2,3)$. These studies together support an active role of the immune system against tumors and suggest that IFN- $\gamma$ is one of the most important players in this process, in part by modulating the immunogenicity of the tumor cells (1-3). IFN- $\gamma$ induces the expression of several components of the antigen-processing machinery,

Received for publication March 29, 2002, and accepted in revised form September 10, 2002.

Address correspondence to: Rolf Kiessling, Cancer Center Karolinska, R8:01, Karolinska Hospital, S-17176 Stockholm, Sweden. Phone: 46-8-51776857; Fax: 46-8-309195;

E-mail: rolf.kiessling@mtc.ki.se.

Conflict of interest: The authors have declared that no conflict of interest exists.

Nonstandard abbreviations used: T cell receptor (TCR); NK receptor (NKR); killer cell Ig-like receptor (KIR); leukocyte Ig-like receptor-1 (LIR-1); short-term ovarian carcinoma cell line (OVAC); tumor-associated lymphocyte (TAL); lymphokine activated killer (LAK); Ig-like transcript-2 (ILT-2); lymphoblastoid cell line (LCL); peptide IVTDFSVIK (IVT); Epstein-Barr virus nuclear antigen-4 (EBNA4); HLA-G signal peptide (Gsp). leading to enhanced presentation of peptides in the context of HLA class I molecules on the cell surface (4). This facilitates the $T$ cell receptor-mediated (TCR-mediated) recognition of target cells by $\mathrm{CD}^{+} \mathrm{T}$ cells. In contrast, IFN- $\gamma$-mediated upregulation of certain HLA class I alleles decreases target cell sensitivity to the cytolytic activity of NK cells due to the engagement of corresponding inhibitory receptors on these cells (5).

Two major classes of HLA class I binding NK receptors (NKRs) have been identified. One class of receptors belongs to the Ig-like superfamily: the killer cell Ig-like receptors (KIRs) and leukocyte Ig-like receptor-1 (LIR-1), which is also known as Ig-like transcript-2 (ILT-2) and CD85j. The second class comprises heterodimers formed by the C-type lectin-like molecules CD94 and NKG2A, -C, or -E $(6,7)$. The ligands for the KIRs are the classical HLA class I molecules HLA-A, -B, and -C. LIR-1 recognizes several HLA class I alleles but also the nonclassical class I molecule HLA-G (7). HLA-G is mainly expressed at the maternofetal interface during pregnancy, where it is thought to downmodulate the maternal immune responses against the semiallogeneic fetus (8). Ectopic expression of this molecule has been re-ported in malignancies including melanoma, renal carcinoma, and breast carcinoma, possibly reflecting a mechanism for the tumor to avoid recognition by the host immune system $(9-11)$. The major ligand for CD94/NKG2 is HLA-E $(7,12-14)$. 
HLA-E expression depends on its binding to nonamer peptides derived from the leader sequences of certain HLA class I molecules $(15,16)$. Thus, HLA-E expression reflects the amount of classical class I molecules expressed by the cell. The interaction of HLA-E with CD94/NKG2 heterodimers results in delivery of inhibitory (NKG2A) or triggering signals (NKG2C, NKG2E), depending on the NKG2 subtype (17). NKRs are also expressed on $\mathrm{T}$ cells, in particular on those with an activated/memory phenotype (18). The functional role of NKR expression on CTLs remains unclear, but several observations suggest that they may inhibit antigen-specific effector function in human CTLs (19-21). Furthermore, the CD94/NKG2A receptors were recently demonstrated to regulate antiviral $\mathrm{CD} 8^{+} \mathrm{T}$ cell responses in mice (22). It was emphasized that IFN- $\gamma$, released during the viral response, may induce expression of $\mathrm{Qa}-1$, the murine orthologue to HLA-E (23), and thereby promote signalling through CD94/NKG2 receptors. To date, there is no experimental evidence for reduced CTL activity as a result of IFN- $\gamma$ modulation of targets.

Here we show that IFN- $\gamma$-treated short-term ovarian carcinoma cell lines (OVACs) were protected from the cytolytic activity of HLA allospecific and peptide-specific $\mathrm{CD}^{+} \mathrm{T}$ cells. This protection was dependent on the engagement of inhibitory CD94/NKG2A receptors expressed on the effector cells. IFN- $\gamma$ treatment of OVACs was found to upregulate the expression of HLA-E mRNA, and data is presented supporting a role for the HLA-G leader sequence-derived signal peptide (Gsp) in the IFN- $\gamma$-mediated protection of OVACs from CTL lysis.

\section{Methods}

Cell lines. Ascites was collected during primary surgery of patients with suspected ovarian carcinoma. Diagnosis was determined by histological examination of the tumor specimens. All were epithelial ovarian carcinomas except one that was a tumor of the fallopian tube. None of the patients had received previous treatment with radio- or chemotherapy. Tumor cells were isolated as previously described (24) and cultured for 2-4 weeks in complete medium, consisting of Iscove's Modified Dulbecco's Medium (Life Technologies Inc., Gaithersburg, Maryland, USA) supplemented with 5-10\% heat-inactivated FCS, $2 \mathrm{mM}$ L-glutamine, $100 \mathrm{IU} / \mathrm{ml}$ penicillin, and 100 $\mu \mathrm{g} / \mathrm{ml}$ streptomycin (Life Technologies Inc.). OVACs were characterized as tumor cells either by the expression of the epithelial cell marker Ber-EP4 or by lack of expression of the monocyte and lymphocyte markers CD3, CD5, CD14, CD16, CD19, and CD56 (25). In some cases, FACS sorting of Ber-EP4-positive cells was performed to enrich for tumor cells. Prior to experiments, tumor cells were treated with $500 \mathrm{IU} / \mathrm{ml}$ of IFN- $\gamma$ (Boehringer Ingelheim International GmbH, Ingelheim, Germany) for 48 hours in complete medium. Tumor-associated lymphocyte (TAL) cultures were established from ascites by first separating cells using Ficoll-Hypaque (Amersham Pharmacia Biotech, Uppsala, Sweden) and then culturing them in AIM-V (Life Technologies Inc., Paisley, United
Kingdom) supplemented with $100 \mathrm{IU} / \mathrm{ml}$ of IL-2 (PeproTech Inc., Rocky Hill, New Jersey, USA). K562 is a human HLA class I-negative erythroleukemia cell line that was kindly provided by G. Nolan (Stanford University, Palo Alto, California, USA). DFW and CKMel-1 are melanoma cell lines that were established at the Immune and Gene Therapy Laboratory of Karolinska Institutet. C1R/A11 is an HLA-A11 transfectant of the C1R lymphoblastoid cell line (LCL), which has a deletion in the HLA class I gene. The ovarian carcinoma cell line Caov- 4 and the breast carcinoma cell line MDA 231 were from American Type Culture Collection (Rockville, Maryland, USA). The human HLA class I-low LCL 721.221 was transfected with HLA-G1 to produce the $721.221 / \mathrm{G} 1$ cell line. Both have been described previously (26).

CTL lines and clones. The CD8 ${ }^{+}$HLA-A11-restricted CTL clone BK289 specific for peptide IVTDFSVIK (IVT), derived from Epstein-Barr virus nuclear antigen-4 (EBNA4), was generated by stimulating PBMCs from an HLA-A11-positive donor with an autologous LCL expressing the IVT peptide and was previously characterized (27). The HLA-A11-specific CTL clone KV109 was generated by stimulating PBMCs from an HLA-A11-negative donor with an irradiated HLA-A11-positive LCL. The specificity of this clone was tested numerous times on a panel of HLA-A11 ${ }^{+}$and HLA-A11- LCLs and phytohemagglutinin blasts as well as on the C1R and C1R/A11 cell lines. The CTL clones (HA-BK clone 2 and clone 8) and the CTL lines (369-11 and 369-26) recognizing HLA-A2 were produced by stimulating PBMCs from HLA-A2-negative donors with irradiated HLA-A2-positive PBMCs. RF1 is a polyclonal tumor-reactive CTL line that recognizes OVAC 19 in an MHC class I-restricted manner and shows no cytolytic activity against K562, C1R, C1R/A2, or 721.221.

HLA-G leader sequence peptide and peptide pulsing experiments. The Gsp (VMAPRTLFL) was purchased from Research Genetics (Huntsville, Alabama, USA). Untreated OVACs were incubated with Gsp or control peptide at $26^{\circ} \mathrm{C}$ for $15-20$ hours in serum-free AIM-V (Life Technologies Inc.). Cells were then harvested, washed in PBS, and used in cytotoxicity assays as described below.

Total RNA preparation and first-strand cDNA synthesis. Total RNA was prepared from OVACs and Caov-4 using RNA Bee according to the manufacturer's protocol (TelTest Inc., Friendswood, Texas, USA). This was followed by first-strand cDNA synthesis using Moloney murine leukemia virus reverse transcriptase as described by the manufacturer (Life Technologies Inc.).

Real-time quantitative PCR. Real-time quantitative PCR was performed to quantify the expression of the HLA-E gene using the ABI Prism 7700 Sequence Detection System (Applied Biosystems, Foster City, California, USA) as previously described (28). Briefly, primers were designed for conserved regions of the HLA-E gene to cover detection of all different HLA-E alleles. The sense primer was 5'-GGGACACCGCACAGATTTT-3' from exon 2 , and the antisense primer was $5^{\prime}$-CTCAGAGGCATCATTTGACTTTT-3' from exon 3, leading to an amplicon of $254 \mathrm{bp}$ in length. The TaqMan probe (FAM-AGAGC- 
GAGGCCGGGTCTCA-TAMRA) (CyberGene AB, Huddinge, Sweden) was designed to span related exon junctions (exons 2 and 3 ) and was labelled with blue 6-FAM (CyberGene AB) as reporter and TAMRA (CyberGene $\mathrm{AB})$ as quencher. The specificity of primers was confirmed by cloning the PCR product into pGEM-T Easy Vector (Promega Corp., Madison, Wisconsin, USA) followed by sequencing using T7 and SP6 primers (CyberGene $A B)$. The relative quantification of HLA-E gene expression was calculated using $\beta$-actin gene expression as endogenous control as previously described (29).

Western blot analysis of HLA-G. All procedures were performed using the Multiphor II Electrophoresis System and ExcelGel SDS homogeneous precast gels (Amersham Pharmacia Biotech, Piscataway, New Jersey, USA). Cells cultured in complete medium or in the presence of IFN- $\gamma(500 \mathrm{IU} / \mathrm{ml})$ for 48 hours at $37^{\circ} \mathrm{C}$ were pelleted by centrifugation and lysed in electrophoresis sample buffer. Aliquots of total cell lysates corresponding to $5 \times 10^{4}$ cells were separated by SDS-PAGE followed by transfer onto nitrocellulose membranes (Millipore $A B$, Sundbyberg, Sweden). Membranes were blocked in PBS containing 5\% milk and $0.1 \%$ Tween-20 and probed with the anti-HLA-G mouse mAb MEM-G/1 (EXBIO Praha AS, Praha, Czech Republic) at a concentration of 1 $\mu \mathrm{g} / \mathrm{ml}$. After incubation with the secondary anti-mouse $\mathrm{Ab}$ conjugated with horseradish peroxidase (Amersham Pharmacia Biotech), membranes were extensively washed and the reaction was visualized by enhanced chemiluminescence according to the manufacturer's protocol (Amersham Pharmacia Biotech). Equal loading of total cell lysates from untreated and IFN- $\gamma$-treated targets was monitored by Western blot analysis of $\beta$-actin using mouse anti- $\beta$-actin $\mathrm{mAb}$ (clone AC-74) purchased from Sigma-Aldrich (St. Louis, Missouri, USA). $A b$ 's and flow cytometry. The following $\mathrm{mAb}$ 's were used: 87G (anti-HLA-G1) was kindly provided by D. Geraghty (Fred Hutchinson Cancer Research Center, Seattle, Washington, USA). HP3D9 (anti-CD94) was kindly provided by M. Lopez-Botet (University Pompeu Fabra, Barcelona, Spain). Z199 recognizes the inhibitory heterodimer CD94/NKG2A and was previously described (30). DAK-G05 (IgG2a isotype control), UCHT1 (anti-CD3), DK23 (anti-CD5), TUK4 (anti-CD14), DJ130 (antiCD16), MOC-1 (anti-CD56), and the R-phycoerythrin-conjugated rabbit anti-mouse IgG were purchased from DAKOPATTS AB (Glostrup, Denmark). The FITCconjugated anti-epithelial antigen Ber-EP4 was from DAKOPATTS AB. The HB-95 (anti-HLA-A, -B, -C), HB-164 (anti-HLA-A11), and HB-54 (anti-HLA-A2) hybridomas were purchased from American Type Culture Collection. Immunofluorescence stainings were done using standard protocols. For HLA-G staining of freshly isolated ovarian tumor cells, an extra step of Fcreceptor blocking was included. Cells were incubated for 1 hour at $4^{\circ} \mathrm{C}$ with $10 \%$ human $\mathrm{AB}$ sera. Isotype-matched control $\mathrm{Ab}$ 's were used as negative control in all stainings. Cells were analyzed on a FACScan flow cytometer (Becton, Dickinson and Co., San Jose, California, USA).
Cytotoxicity assays and blocking experiments. Standard ${ }^{51} \mathrm{Cr}$-release assays were performed. Briefly, target cells, either untreated or treated with IFN- $\gamma$ for 48 hours, were labelled with $\mathrm{Na}^{51} \mathrm{CrO}_{4}\left(0.1 \mu \mathrm{Ci} / 10^{6}\right.$ cells; Amersham Pharmacia Biotech) at $37^{\circ} \mathrm{C}$ for 1 hour. For peptide-specific recognition, targets were incubated with 1 $\mu \mathrm{g} / \mathrm{ml}$ of peptide during labelling. After extensive washing, target cells were incubated with effectors at different effector-to-target ratios in triplicate for 4 hours at $37^{\circ} \mathrm{C}$. Supernatants were harvested and radioactivity was determined using a $\gamma$ counter (Wallac AB, Upplands Väsby, Sweden). In blocking experiments, the effector cells were preincubated with $10 \mu \mathrm{g} / \mathrm{ml}$ of MOC-1 (anti-CD56) or HP3D9 (anti-CD94) mAb at $4^{\circ} \mathrm{C}$ for 30 minutes prior to addition to the labelled target cells in a 4-hour ${ }^{51} \mathrm{Cr}$-release assay.

\section{Results}

IFN- $\gamma$ protects tumor cells from lysis by CTLs. We assessed the overall ability of short-term primary tumor cell lines to serve as targets for CTL-mediated immune effector functions upon IFN- $\gamma$ treatment. To this end we used HLA class I-specific $\mathrm{CD}^{+} \mathrm{T}$ cells recognizing the targets regardless of the endogenous processing and presentation of one particular epitope. Unexpectedly, IFN- $\gamma$-treated OVAC 16 (which is HLA-A11+) was protected from lysis by an HLA-A11-alloreactive $\mathrm{CD}^{+} \mathrm{T}$ cell clone, KV109 ( $P=0.01$; Figure 1a). K562 was not killed by KV109 excluding nonspecific, NK-like activity by the CTLs. Next we used a high-affinity $T$ cell clone specific for an HLA-A11-restricted EBNA4-derived epitope (IVT) as effector cells against peptide-pulsed HLA-A11-positive OVAC 16. This revealed that peptide-specific lysis was also inhibited by IFN- $\gamma$ treatment of this OVAC $(P<0.01$; Figure $1 \mathrm{~b})$. In contrast, the recognition of a peptidepulsed HLA-A11-transfected LCL line (C1R/A11) was not affected by IFN- $\gamma$ treatment (Figure 1c). IFN- $\gamma$-mediated protection of the OVAC was observed at all peptide concentrations tested $(P<0.01$; Figure $1 \mathrm{~d})$. A tenfoldhigher concentration of the peptide was required to achieve similar levels of lysis of the IFN- $\gamma$-treated OVAC 16 when compared with untreated OVAC 16 . Furthermore, maximal lysis of IFN- $\gamma$-treated OVAC 16 was approximately $50 \%$ of the level observed with untreated cells. IFN- $\gamma$-treated OVAC 16 was also protected from lysis by autologous TALs $(P<0.01$; Figure $1 \mathrm{e})$. However, these TALs displayed lymphokine-activated killer (LAK) activity as determined by the lysis of K562. TAL cultures consisted predominantly of T cells (mean, $87 \%$ ), but a varying percentage of NK cells was usually present in these cultures. In order to evaluate whether OVACs in general gained resistance to CTL lysis after IFN- $\gamma$ treatment, we established several HLA-A2 ${ }^{+}$OVACs. IFN- $\gamma$ treatment of all nine tested cell lines resulted in strong protection from lysis by a panel of different HLAA2-restricted CTLs (Table 1). In contrast, IFN- $\gamma$-treated melanoma cell lines (DFW and CKMel-1) and a breast cancer line (MDA 231) were killed at similar or higher levels than the untreated tumors. Also, the long-term, in 
vitro-propagated ovarian carcinoma cell line Caov-4 was killed more efficiently after IFN- $\gamma$ treatment. In conclusion, these results showed that all IFN- $\gamma$-treated OVACs were protected from CTLs, in contrast to long-term established cell lines of different tumor origin. These results were not due to decreased viability or function of CTLs imposed by the tumors, since $\mathrm{T}$ cells that were cocultured with IFN- $\gamma$-treated, HLA-mismatched OVACs remained fully capable of killing a third-party cell line (data not shown). Furthermore, there was no evidence for a general resistance to apoptosis or cell death in IFN- $\gamma$ treated OVACs, as these cells were more sensitive to Fasmediated killing induced by an anti-Fas Ab when compared with untreated OVACs (data not shown).

IFN- $\gamma$ treatment results in increased expression of total HLA class I, HLA-A2, and HLA-A11. IFN- $\gamma$ is known to upregulate the expression of several components of the antigen processing and presentation machinery, leading to enhanced stability and expression of HLA class I at the cell surface. Although we observed paradoxical protection from CTL-mediated lysis when tumor cells were treated with IFN- $\gamma$, we found no evidence of altered regulatory effects by IFN- $\gamma$ on the expression of HLA class I molecules. The surface expression of total HLA class I, HLA-A2, and HLA-A11 was significantly increased by IFN- $\gamma$ treatment for all OVACs in this study, as exemplified by OVAC 16 in Figure 2.

Blockade of CD94/NKG2A inhibitory receptors restored killing of IFN- $\gamma$-treated tumor cells. It has become evident that NK cell receptors are expressed on $\mathrm{T}$ cells, in particular on those with an activated/memory phenotype (18). Therefore, we studied the role of inhibitory receptors in the IFN- $\gamma$-mediated protection of OVACs. Having observed this phenomenon in a high number of distinct effector-target combinations (Table 1), we thought it unlikely that the protection was due to the recognition of a particular HLA allele by its corresponding inhibitory receptor. Instead, we focused on the CD94/NKG2A receptor, as this receptor may survey a broader panel of HLA class I alleles due to its recognition of HLA-E (7). All our tested $\mathrm{T}$ cell clones and lines expressed the inhibitory heterodimer CD94/NKG2A, as determined by the mAb Z199, which specifically recognizes CD94/NKG2A (30) (data not shown). When preincubating various effector cell populations, including the HLA-A11-specific $\mathrm{CD}^{+} \mathrm{T}$ cell clone KV109 (Figure 3a) and peptide-specific BK289 (Figure 3b), with an anti-CD94 mAb (HP3D9), the protective effect of IFN- $\gamma$ was abolished $(P<0.01$ and $P<0.05$, respectively). The recognition of IFN- $\gamma$-treated OVAC 19 by the tumor-reactive T cell line RF1 was also partially restored $(50 \%)$ in the presence of HP3D9 $(P<0.05$; Figure $3 \mathrm{c})$. As control, T cells were preincubated with an isotype-matched Ab against CD56, without significant impact on the recognition of tumor targets. Early reports have described that LAK cell killing of tumors, similar to the TAL-mediated killing of OVACs presented in this study, may be hampered by IFN- $\gamma(31)$, but the role of CD94/NKG2A in this phenomenon was never investigated. Figure $3 \mathrm{~d}$ demonstrates a total restoration of the lysis of IFN- $\gamma$-treated, autologous short-term tumor cells in the presence of the anti-CD94 $\mathrm{mAb}$. It has been reported that $\mathrm{CD}^{+} \mathrm{T}$ cells that are cultured in IL-2 may display NK-like activity, bypassing the need for TCR-mediated stimulation, and that this non-
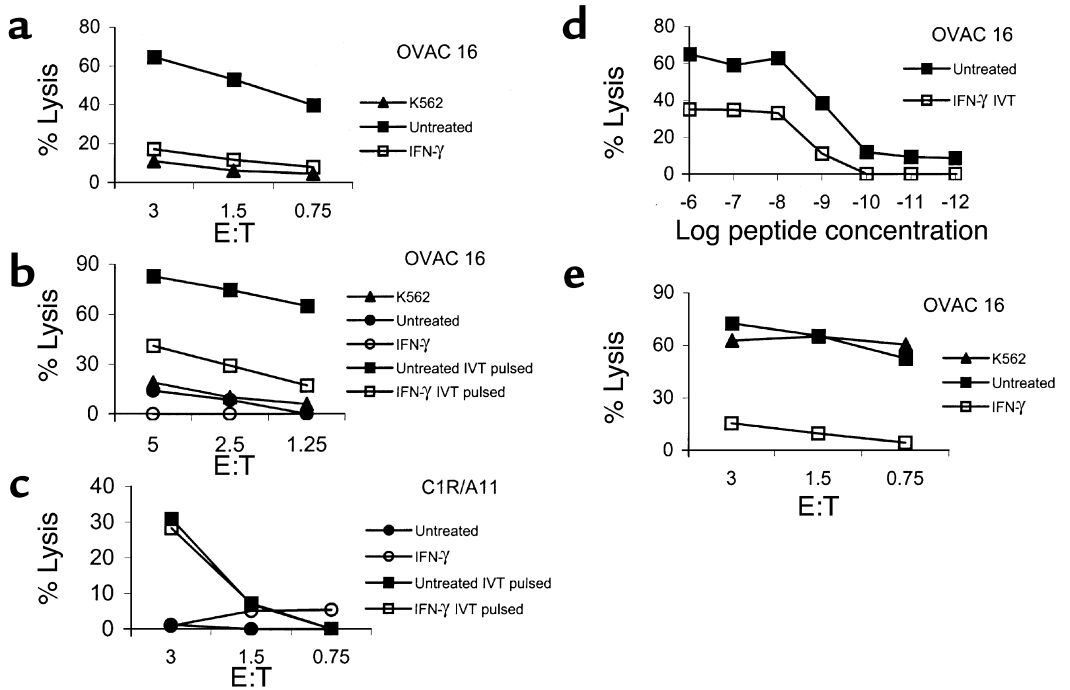

\section{Figure 1}

IFN- $\gamma$ protects short-term ovarian carcinomas from CTL lysis. The effect of IFN- $\gamma$ on CTL recognition of OVACs was evaluated in 4-hour ${ }^{51} \mathrm{Cr}$-release assays. (a) K562 and untreated or IFN- $\gamma$-treated OVAC 16 (HLA-A11 ${ }^{+}$) were used as targets for the HLA-A11-specific, CD8 ${ }^{+}$ CTL clone KV109. (b) OVAC 16 was untreated or treated with IFN- $\gamma$ and then pulsed or not pulsed with the HLA-A11-restricted, EBNA4derived epitope IVT at a concentration of $1 \mu \mathrm{g} / \mathrm{ml}$ and used as a target for the IVT-specific, CD8 ${ }^{+}$T cell clone BK289. (c) C1R/A11 was untreated or treated with IFN- $\gamma$ and then pulsed or not pulsed with the IVT peptide $(1 \mu \mathrm{g} / \mathrm{ml})$ and used as a target for BK289. (d) Untreated or IFN- $\gamma$-treated OVAC 16 was prepulsed with IVT peptide at concentrations ranging from $10^{-12}$ to $10^{-6} \mathrm{~g} / \mathrm{ml}$ and used as a target for BK289 at an effector-to-target ratio of 3:1. Recognition of non-pulsed targets was below 7\%. Results from one of at least three experiments are shown. (e) K562 and untreated or IFN- $\gamma$-treated OVAC 16 was used as a target for autologous TALs. 
OVAC 16

HLA class I

HLA-A2

HLA-A11

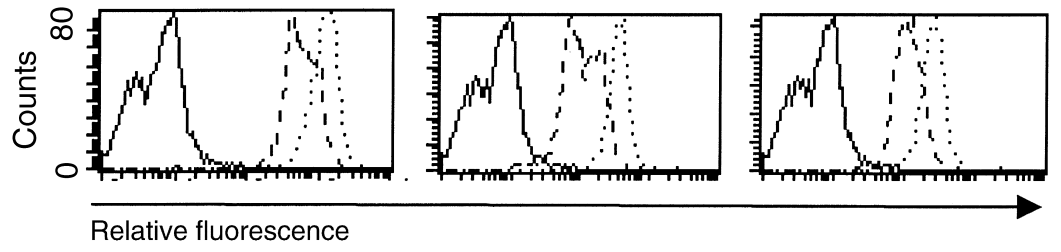

Figure 2

Intact regulatory function of IFN- $\gamma$ on HLA class I expression in OVACs. OVAC 16 was untreated or treated with IFN- $\gamma$ for 48 hours and then stained for the expression of total HLA class I, HLA-A2, and HLA-A11. Stainings of untreated cells (dashed line) and IFN- $\gamma$-treated cells (dotted line) are shown. Isotype control stainings are depicted as a solid line. Histograms from one representative staining out of three of this particular tumor is shown.

specific lysis is dampened by inhibitory receptors expressed on the T cells (32). However, this was not the case for the peptide-specific T cell clone BK289 (Figure $3 \mathrm{~b})$, as non-peptide pulsed targets were not killed even in the presence of anti-CD94 $\mathrm{Ab}$ (data not shown). We therefore concluded that IFN- $\gamma$ protected OVACs by enhancing the negative signalling via the CD94/NKG2A receptor.

Expression of nonclassical class I molecules. HLA-E mRNA expression by OVACs was evaluated by real-time quantitative PCR. We show that IFN- $\gamma$-treated OVACs upregulated their expression of HLA-E mRNA (Figure 4a). However, there was no difference in the regulation of HLA-E between OVACs and the long-term in vitro-propagated cell line Caov-4 (Figure 4a). Nor was there any correlation between the extent of upregulation of HLA-E in a particular cell line and the IFN- $\gamma$-mediated protection of this tumor (data not shown). These results demonstrated that the degree of HLA-E mRNA expression could not be the only factor determining whether or not tumors were protected by IFN- $\gamma$. As HLA-G expression, by binding of the HLA-G signal peptide (Gsp) to the HLA-E molecule, may protect target cells from lysis by CD94/NKG2A-expressing effector cells (33), we investigated the expression pattern of this nonclassical HLA molecule on ovarian tumor cells. HLA-G expression was assessed by FACS analysis of 17 freshly isolated tumor samples from patients with ovarian carcinoma. Nine of 17 samples (53\%) were positive for HLA-G as determined by the $\mathrm{MAb} 87 \mathrm{G}$, which is specific for HLA-G1 (34). In Figure 4b, three examples of HLA-G1-positive tumor samples are shown, together with the staining of the negative (721.221) and positive (721.221/G1) controls. Double staining for Ber-EP4 and

Table 1

IFN- $\gamma$ protects a broad panel of OVACs from lysis by several HLA-A2-restricted effectors

\begin{tabular}{|c|c|c|c|c|c|c|}
\hline \multicolumn{2}{|c|}{ Effector } & \multicolumn{2}{|c|}{ Target } & \multicolumn{3}{|c|}{ Cytotoxic assay } \\
\hline Name & Phenotype & Name & HLA-A $2^{A}$ & $E / T^{B}$ & $\%$ lysis NTC & $\%$ lysis IFN \\
\hline HA-BK clone 2 & CD4 & OVAC 6 & + & $3: 1$ & 40 & 16 \\
\hline $369-11$ & $\mathrm{CD} 4 / \mathrm{CD} 8$ & OVAC 10 & + & $5: 1$ & 50 & 17 \\
\hline $369-26$ & CD8 & OVAC 10 & + & $5: 1$ & 70 & 47 \\
\hline $369-11$ & $\mathrm{CD} 4 / \mathrm{CD} 8$ & OVAC 11 & + & $5: 1$ & 55 & 8 \\
\hline $369-26$ & CD8 & OVAC 11 & + & $5: 1$ & 64 & 27 \\
\hline HA-BK clone 8 & CD8 & OVAC 11 & + & $13: 1$ & 46 & 34 \\
\hline $369-11$ & $\mathrm{CD} 4 / \mathrm{CD} 8$ & OVAC 12 & + & $5: 1$ & 37 & 5 \\
\hline $369-26$ & $\mathrm{CD} 8$ & OVAC 12 & + & $5: 1$ & 47 & 12 \\
\hline $369-26$ & CD8 & OVAC 12 & + & $3: 1$ & 41 & 16 \\
\hline $369-11$ & $\mathrm{CD} 4 / \mathrm{CD} 8$ & OVAC 13 & + & $5: 1$ & 42 & 5 \\
\hline $369-26$ & CD8 & OVAC 13 & + & $5: 1$ & 60 & 18 \\
\hline HA-BK clone 8 & CD8 & OVAC 13 & + & $13: 1$ & 40 & 10 \\
\hline $369-11$ & $\mathrm{CD} 4 / \mathrm{CD} 8$ & OVAC 14 & + & $5: 1$ & 35 & 3 \\
\hline $369-26$ & $\mathrm{CD} 8$ & OVAC 14 & + & $5: 1$ & 57 & 41 \\
\hline HA-BK clone 2 & CD4 & OVAC 16 & + & $3: 1$ & 45 & 32 \\
\hline $435-26$ & CD8 & OVAC 24 & + & $10: 1$ & 30 & 14 \\
\hline $435-26$ & CD8 & OVAC 33 & + & $10: 1$ & 39 & 20 \\
\hline HA-BK clone 2 & CD4 & Caov-4 & + & $3: 1$ & 29 & 42 \\
\hline HA-BK clone 2 & CD4 & MDA 231 & + & $3: 1$ & 23 & 30 \\
\hline HA-BK clone 2 & CD4 & CK-Mel 1 & + & $3: 1$ & 23 & 24 \\
\hline $369-26$ & CD8 & DFW & + & $10: 1$ & 5 & 17 \\
\hline
\end{tabular}

Different effectors were used in cytotoxic assays against a broad panel of untreated (NT) and IFN-g-treated (IFN) tumor cell lines. Effectors and targets are further described in Methods. Targets used include the OVACs, the melanoma cell lines CKMel-1 and DFW, the breast cancer cell line MDA 231, and the OVAC Caov-4. AHLA-A2 positivity as determined by the staining of tumor cells with the HB-54-derived mAb. ${ }^{B} \mathrm{E} / \mathrm{T}$, effector-to-target ratio. ${ }^{\mathrm{C} P e r c e n t}$ lysis was determined in 4-hour $51 \mathrm{Cr}$-release assays as described in Methods. 
HLA-G confirmed expression of HLA-G by tumor cells (data not shown). Surface expression of HLA-G was gradually lost upon in vitro culture of the short-term tumor cell lines. However, the expression of HLA-G could still be detected in total cell lysates of all OVACs, as determined in Western blot assays (Figure 4c). Furthermore, HLA-G expression was increased by IFN- $\gamma$. In contrast, no expression of HLA-G was found in the long-term ovarian carcinoma cell line Caov-4 (Figure 4c). These results demonstrated a correlation between the upregulation of HLA-G and the protective effect of IFN- $\gamma$.

Role of the HLA-G leader sequence-derived peptide. To further investigate the role of HLA-G, we preincubated OVAC 19 with the HLA-G leader sequence-derived peptide at different concentrations. Gsp, but not an HLA class I-binding HIV peptide, mediated protection from killing by the CD94/NKG2A-expressing tumor-reactive CTL line RF1 (Figure 5). Thus, protection of OVACs from CTL lysis could be induced by addition of the HLA-G leader sequence peptide to the tumor cells.

\section{Discussion}

The data presented in this study point to a potential adverse effect that IFN- $\gamma$ may have on T cell-mediated recognition of tumor cells. We demonstrate that IFN- $\gamma$-treated OVACs are protected from HLA-A11specific (Figure 1a) and peptide-specific (Figure 1b) lysis by $\mathrm{CD}^{+} \mathrm{T}$ cells. This protection was due to
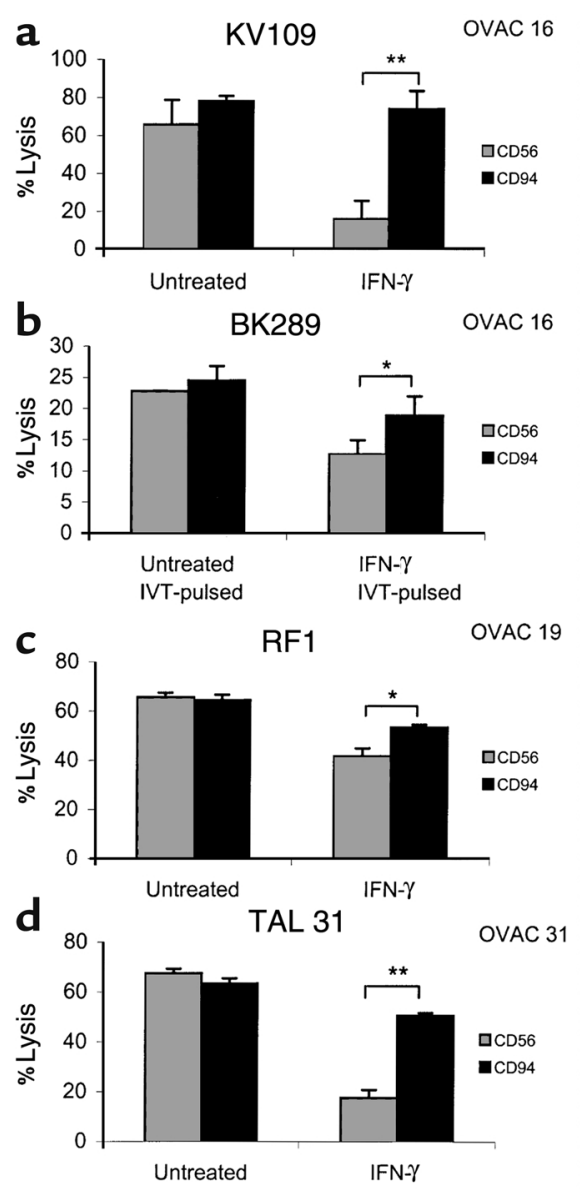

enhanced inhibitory signalling via CD94/NKG2A receptors expressed on the effector cell populations, as it was abrogated by blockade of CD94 (Figure 3). These results reveal that IFN- $\gamma$ may shift the balance of triggering and inhibitory signals, leading to a situation in which $T$ cells are incapable of killing otherwise sensitive HLA class I-expressing targets. Thus, the increased inhibitory signalling via CD94/NKG2A seems more important to the CTLs than does the upregulation of the positively signalling ligand to the TCR.

It is becoming evident that NKRs, although originally described on NK cells, are also expressed and have important functions on T cells (18). In healthy humans and mice, CD94/NKG2 receptors are expressed by less than $5 \%$ of $\mathrm{CD}^{+} \mathrm{T}$ cells (35). However, it has been demonstrated that the CD94/NKG2A receptor is upregulated in activated $\mathrm{CD}^{+} \mathrm{T}$ cells, either as a result of TCR-mediated stimulation or from cytokine stimulation by TGF- $\beta$ or IL-15 $(36,37)$. All CTL clones that were used in this study expressed CD94/NKG2A, and some also coexpressed other NKRs, including LIR-1 and the KIR molecules KIR2DL2 and KIR3DL2 (data not shown). Additionally, in the TAL cultures, both CD94/NKG2A and LIR-1 were frequently expressed, mainly on $\mathrm{CD}^{6} 6^{+}$, CCR7-, $\mathrm{CD}^{2} 5 \mathrm{RA}^{+}$, and $\mathrm{CD}^{+} \mathrm{T}$ cells (unpublished observations) corresponding to the terminally differentiated effector cells (38). Therefore, it cannot be excluded that NKRs other than CD94/NKG2A contributed to the IFN- $\gamma$ mediated protection of OVACs. However, this contribution is unlikely to play a major role, as it would not be affected by the blockade of CD94/NKG2A receptors.

There are several theories to explain why $T$ cells express inhibitory receptors (18). They may fine-tune the T cell responses by raising the threshold for TCR triggering (39), thereby serving as a mechanism for peripheral tolerance (40). It has also been described that KIR-expressing $T$ cells are more resistant to activation-induced cell death, and thus that KIR molecules are involved in the development of long-term memory $\mathrm{T}$ cells (41). The CD94/NKG2A receptor inhibits cytokine production and cytolytic activity of antigen-specific $T$ cells (20). In a recent study it was demonstrated that the inability of antiviral $\mathrm{CD}^{+} \mathrm{T}$ cells to control virus-induced oncogenesis was due to the upregulation of CD94/NKG2A receptors on the T cells (22). Thus, the negative signal provid-

\section{Figure 3}

Blockade of CD94/NKG2A inhibitory receptors restores killing of IFN- $\gamma$-treated OVACs. The effect of anti-CD56 (MOC-1) or antiCD94 (HP3D9) mAb's on the IFN- $\gamma$-mediated protection of OVACs was evaluated in the following situations: The HLA-A $11^{+}$ OVAC 16 was used as target for the HLA-A11-specific T cell clone KV109 (a) and for the IVT-specific T cell clone BK289 (b). The peptide concentration in $\mathbf{b}$ was $1 \mu \mathrm{g} / \mathrm{ml}$. Recognition of unpulsed targets by BK289 was below 5\% and is not shown. (c) The HLA class I-restricted, tumor-reactive CTL line RF1 was used as an effector against untreated and IFN- $\gamma$-treated OVAC 19. (d) Reactivity of TAL 31 against autologous OVAC 31. Error bars represent SD of triplicate cultures. ${ }^{*} P<0.05,{ }^{*} P<0.01$ as determined by twotailed Student $t$ test. 
a

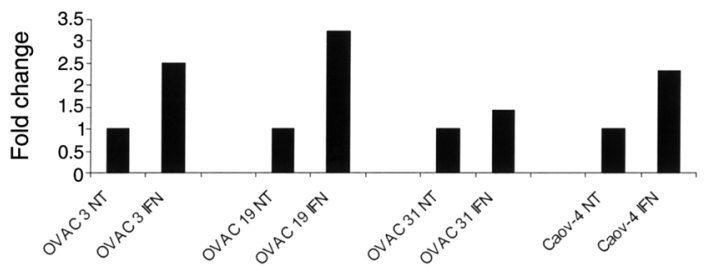

b

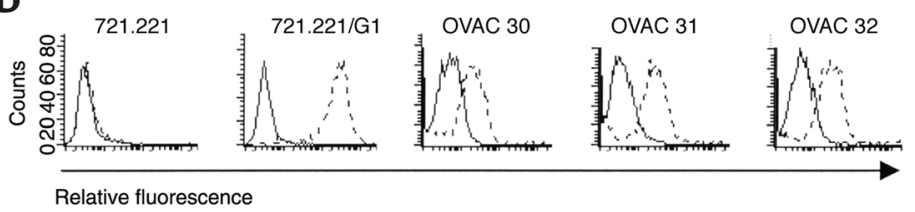

c

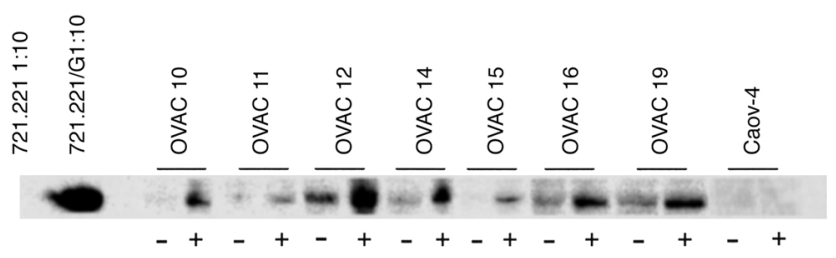

\section{Figure 4}

Expression of nonclassical HLA molecules. (a) The expression of HLA-E mRNA was evaluated in indicated OVACs by real-time quantitative PCR assay. Results are presented as fold increase in IFN- $\gamma$-treated cells (IFN) compared with untreated cells (NT). Results from one of two experiments are shown. (b) The expression of HLA-G by freshly isolated ovarian tumor cells was monitored by flow cytometry analysis. Indicated cells were stained with isotype control (mouse IgG2a) (solid line) or anti-HLA-G1 (87G) (dotted line). Results from one representative staining of at least ten are shown. (c) Western blot analysis of HLA-G expression in indicated OVACs and Caov-4 was performed using the HLA-G-specific mAb MEM-G/ 1 at a concentration of $1 \mu \mathrm{g} / \mathrm{ml}$. The LCLs 721.221 and 721.221 transfected with HLA-G1 (721.221/G1) were used as positive and negative controls. These were diluted 1:10 because of the excess amount of HLA-G expressed by the $721.221 / G 1$ transfectant. Cell extracts of untreated and IFN- $\gamma$-treated samples were loaded in equal amounts as determined by Western blots for $\beta$-actin (data not shown). ed via the ligand-NKR interaction may dominate over the triggering signal via HLA class I and the TCR. However, in most human viral and tumor systems, this inhibitory effect was only partial, as it could be overcome by increasing the concentrations of peptide epitopes on the target cells $(39,42)$. In the present study, CD94/NKG2A-mediated protection was observed at all peptide concentrations tested (Figure 1d), indicating that the quality of the inhibitory signal rather than the strength of the positive signal determined the cytolytic activity of $\mathrm{T}$ cells.

The major ligand for CD94/NKG2A is HLA-E (7, $12-14)$. HLA-E expression is upregulated by IFN- $\gamma$. This may be due to increased accessibility of leader sequence-derived peptides from other HLA class I molecules, but IFN- $\gamma$-upregulated HLA-E expression was also shown to be mediated at the transcriptional level (43). IFN- $\gamma$, in synergy with the cytomegalovirus protein UL40, protects targets from NK cells via the upregulation of HLA-E (44). Our study represents the first example of IFN- $\gamma$-mediated protection of tumor targets from CD94/NKG2A-expressing $\mathrm{CD}^{+} \mathrm{T}$ cells. This finding may have important biological implications and supports the hypothesis put forward by Moser et al. that IFN- $\gamma$ released during an antiviral or anti-tumoral response may lead to enhanced inhibitory signalling to antigen-specific $\mathrm{T}$ cells carrying the CD94/NKG2A receptors and thereby turn off their effector functions (22).

We also demonstrate, as a new finding, that autologous TALs are incapable of lysing IFN- $\gamma$-treated OVACs due to inhibitory signals provided via CD94/NKG2A receptors. While OVACs were protected from CTL lysis, other tumor cell lines, including the long-term in vitro-propagated ovarian carcinoma cell line Caov-4, were in fact more efficiently killed after IFN- $\gamma$ treatment (Table 1). We speculated that this observation could be explained by upregulation of HLA-E by IFN- $\gamma$ on OVACs but not (or to a lesser extent) on other tumor cell lines. By investi- gating the expression of HLA-E mRNA using a real-time quantitative PCR assay, we demonstrate that IFN- $\gamma$ induces the expression of HLA-E in primary cultures of ovarian tumor cells but also in the long-term ovarian carcinoma cell line Caov-4 (Figure 4a). There was no correlation between the level of HLA-E upregulation and the protective effect of IFN- $\gamma$ on tumor cell lines (data not shown). Due to lack of serological reagents, the role of HLA-E surface expression could not be examined. However, our data indicate that the level of expression of HLA-E that was induced by IFN- $\gamma$ could not alone explain the discrepancy between short-term and long-term tumor cell lines. Binding studies of soluble recombinant HLA-E molecules and soluble CD94/ NKG2A proteins revealed a threshold-like behavior of interaction between HLA-E and CD94/NKG2 (45). It was shown that the nature of the HLA class I leader sequence peptide that was assembled with HLA-E affected the

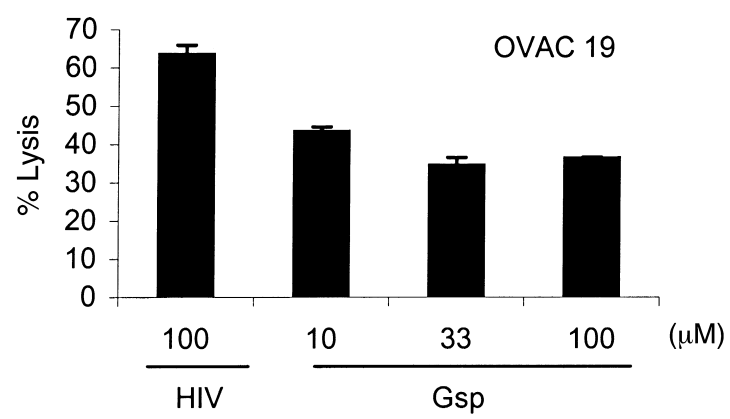

\section{Figure 5}

Role of the HLA-G leader sequence peptide. OVAC 19 was incubated with different concentrations of Gsp as indicated in the figure. HLA-A2-binding HIV peptide (HIV-RT 476-484) was used as negative control at $100 \mu \mathrm{g} / \mathrm{ml}$. HIV- and Gsp-pulsed OVAC 19 was then used as target in a 4-hour ${ }^{51} \mathrm{Cr}$-release assay. The HLA class I-restricted, tumor-specific CTL line RF1 was used as effector. Results from one of two experiments are shown. 
binding of HLA-E to CD94/NKG2A, and that strong binding correlated with the functional inhibitory capacity of the various HLA alleles $(12,13,15,33)$. The leader sequence peptide derived from HLA-G was demonstrated to mediate a particularly strong binding of the HLA-E peptide complex to CD94/NKG2A (45). In fact, it has been suggested that the interaction between HLA-E and CD94/NKG2A represents a nonoverlapping, receptor-ligand system complementary to HLA-G/LIR-1 by which NK cells recognize HLA-G-expressing cells (46).

We report the novel finding of frequent expression of HLA-G on freshly isolated noncultured tumor cells derived from the ascites of patients with ovarian carcinoma (Figure 4b). Surface expression of HLA-G was found in $53 \%$ of the tumor samples, but was gradually lost upon in vitro propagation, although it was still detectable in total cell lysates of all OVACs (Figure 4c). Loss of HLA-G surface expression upon in vitro culture was previously demonstrated in renal carcinoma and suggests that the incidence of HLA-G expression in vivo may be higher than that reported on established tumor cell lines (9). In fact, HLA-G is rarely found in established cell lines (47). This was further supported by our observation that HLA$G$ could not be detected in the long-term ovarian carcinoma cell line Caov- 4 before or after IFN- $\gamma$ treatment by either of the methods used here to detect HLA-G (Figure $4 \mathrm{c}$ and data not shown). It is tempting to speculate that the expression of the HLA-G molecule is dependent on some yet-undefined factor that is present in vivo but lacking during in vitro cultivation. Furthermore, we demonstrate that HLA-G expression in primary cultures of ovarian carcinoma was induced by IFN- $\gamma$ (Figure 4c). Increased intracellular amounts of HLA-G may provide a source of Gsp accessible to the HLA-E molecule. This may lead to strong inhibitory signalling via CD94/NKG2A receptors. Although not formally proven, support for this possibility was provided by the result of the peptide pulsing experiment presented in this study. Preincubation of untreated OVAC 19 with Gsp mimicked the effect of IFN- $\gamma$ and mediated protection from CTL lysis (Figure 5). Such involvement of HLA-G would also explain why primary cultures of ovarian carcinoma were protected by IFN- $\gamma$ while the non-HLA-G expressing, long-term ovarian carcinoma cell line Caov-4 was not.

The capacity of IFN- $\gamma$ to upregulate the expression of HLA class I molecules may be important in situations where the expression of class I is decreased or even totally lost, as has been reported to be the case in several tumors (48). This property of IFN- $\gamma$ has been exploited in clinical trials in which recombinant IFN- $\gamma$, alone or in combination with other cytokines such as IL-2 and GM-CSF, was administrated to patients with malignant tumors $(49,50)$. In a large randomized clinical trial, melanoma patients were treated with recombinant IFN- $\gamma$ without any improvement of overall survival (50). Intraperitoneal administration of recombinant IFN- $\gamma$ to patients with ovarian carcinoma resulted in increased levels of HLA class I molecules on the tumor cells, but no significant clinical improvement was achieved (49). Our finding that IFN- $\gamma$ protects OVACs via a CD94/NKG2A-dependent mechanism might explain why the use of recombinant IFN- $\gamma$ has not been more successful in the clinical setting. The potentially negative effect of administrating recombinant IFN- $\gamma$ alone or as adjuvant to immune therapy interventions should therefore be carefully considered.

In closing, our data demonstrate an important regulatory role of inhibitory NK receptors in CTL-mediated recognition of short-term established OVACs. We have previously shown that IFN- $\gamma$ is selectively expressed in ovarian cancer biopsies (51). Furthermore, we have detected IFN- $\gamma$ in ascites fluid at levels comparable to those used to protect tumor cells in the present study (unpublished observations). Thus, IFN- $\gamma-$ induced expression of ligands for inhibitory NK receptors may constitute a tumor escape mechanism from CTL-mediated immunity. It was recently shown in a murine model that blockade of inhibitory receptors on adoptively transferred NK cells leads to augmentation of their antitumor effect in vivo (52). We suggest that similar approaches could be used to overcome the NKR-mediated inhibition of $\mathrm{T}$ cell-mediated antitumoral responses.

\section{Acknowledgments}

This work was supported by grants from the Swedish Cancer Society, the Cancer Society of Stockholm, the King Gustaf V Jubilee Fund, the European Community, and from a gene therapy grant awarded to R. Kiessling from the Swedish Research Council, the Wallenberg Foundation, the Swedish Cancer Society, and the Swedish Foundation for Strategic Research (Stockholm, Sweden). K.-J. Malmberg was supported by grants from the Swedish Society for Medical Research. C.T. de Matos was supported by a Ph.D. fellowship from Fundação para a Ciência a Tecnologia (PRAXIS XXI/BD/4047/94). V. Levitsky was supported by grants awarded by the Swedish Cancer Society, the Swedish Paediatric Cancer Foundation, and the Karolinska Institutet (Stockholm, Sweden).

1. Shankaran, V., et al. 2001. IFNgamma and lymphocytes prevent primary tumour development and shape tumour immunogenicity. Nature. 410:1107-1111.

2. Kaplan, D.H., et al. 1998. Demonstration of an interferon gammadependent tumor surveillance system in immunocompetent mice. Proc. Natl. Acad. Sci. USA. 95:7556-7561.

3. Dighe, A.S., Richards, E., Old, L.J., and Schreiber, R.D. 1994. Enhanced in vivo growth and resistance to rejection of tumor cells expressing dominant negative IFN gamma receptors. Immunity. 1:447-456.

4. Rock, K.L., and Goldberg, A.L. 1999. Degradation of cell proteins and the generation of MHC class I-presented peptides. Annu. Rev. Immunol. 17:739-779.

5. Moretta, A., et al. 1996. Receptors for HLA class-I molecules in human natural killer cells. Annu. Rev. Immunol. 14:619-648.

6. Long, E.O., and Rajagopalan, S. 2000. HLA class I recognition by killer cell Ig-like receptors. Semin. Immunol. 12:101-108.

7. Lopez-Botet, M., Llano, M., Navarro, F., and Bellon, T. 2000. NK cell recognition of non-classical HLA class I molecules. Semin. Immunol. 12:109-119.

8. Carosella, E.D., Paul, P., Moreau, P., and Rouas-Freiss, N. 2000. HLA-G and HLA-E: fundamental and pathophysiological aspects. Immunol. Today. 21:532-534.

9. Ibrahim, E.C., et al. 2001. Tumor-specific upregulation of the nonclassical class I HLA-G antigen expression in renal carcinoma. Cancer Res. 61:6838-6845. 
10. Lefebvre, S., et al. 2002. Specific activation of the non-classical class I histocompatibility HLA-G antigen and expression of the ILT2 inhibitory receptor in human breast cancer. J. Pathol. 196:266-274.

11. Paul, P., et al. 1999. Heterogeneity of HLA-G gene transcription and protein expression in malignant melanoma biopsies. Cancer Res. 59:1954-1960.

12. Braud, V.M., et al. 1998. HLA-E binds to natural killer cell receptors CD94/NKG2A, B and C. Nature. 391:795-799.

13. Borrego, F., Ulbrecht, M., Weiss, E.H., Coligan, J.E., and Brooks, A.G. 1998. Recognition of human histocompatibility leukocyte antigen (HLA)-E complexed with HLA class I signal sequence-derived peptides by CD94/NKG2 confers protection from natural killer cell-mediated lysis. J. Exp. Med. 187:813-818.

14. Lee, N., et al. 1998. HLA-E is a major ligand for the natural killer inhibitory receptor CD94/NKG2A. Proc. Natl. Acad. Sci. USA. 95:5199-5204.

15. Lee, N., Goodlett, D.R., Ishitani, A., Marquardt, H., and Geraghty, D.E. 1998. HLA-E surface expression depends on binding of TAP-dependent peptides derived from certain HLA class I signal sequences. J. Immunol. 160:4951-4960.

16. Braud, V., Jones, E.Y., and McMichael, A. 1997. The human major histocompatibility complex class Ib molecule HLA-E binds signal sequencederived peptides with primary anchor residues at positions 2 and 9. Eur. J. Immunol. 27:1164-1169.

17. Lopez-Botet, M., et al. 2000. Paired inhibitory and triggering NK cell receptors for HLA class I molecules. Hum. Immunol. 61:7-17.

18. McMahon, C.W., and Raulet, D.H. 2001. Expression and function of NK cell receptors in CD8+ T cells. Curr. Opin. Immunol. 13:465-470.

19. Guerra, N., et al. 2000. Killer inhibitory receptor (CD158b) modulates the lytic activity of tumor-specific $\mathrm{T}$ lymphocytes infiltrating renal cell carcinomas. Blood. 95:2883-2889.

20. Speiser, D.E., et al. 1999. In vivo expression of natural killer cell inhibitory receptors by human melanoma-specific cytolytic $\mathrm{T}$ lymphocytes. J. Exp. Med. 190:775-782.

21. Ikeda, H., et al. 1997. Characterization of an antigen that is recognized on a melanoma showing partial HLA loss by CTL expressing an NK inhibitory receptor. Immunity. 6:199-208.

22. Moser, J.M., Gibbs, J., Jensen, P.E., and Lukacher, A.E. 2002. CD94NKG2A receptors regulate antiviral CD8+ T cell responses. Nat. Immunol. 3:189-195.

23. Vance, R.E., Kraft, J.R., Altman, J.D., Jensen, P.E., and Raulet, D.H. 1998. Mouse CD94/NKG2A is a natural killer cell receptor for the nonclassical major histocompatibility complex (MHC) class I molecule Qa-1(b). J. Exp. Med. 188:1841-1848.

24. Ioannides, C.G., et al. 1991. Tumor cytolysis by lymphocytes infiltrating ovarian malignant ascites. Cancer Res. 51:4257-4265.

25. Rabinowich, H., et al. 1998. Lymphocyte apoptosis induced by Fas ligand- expressing ovarian carcinoma cells. Implications for altered expression of T cell receptor in tumor-associated lymphocytes. J. Clin. Invest. 101:2579-2588.

26. Colonna, M., et al. 1997. A common inhibitory receptor for major histocompatibility complex class I molecules on human lymphoid and myelomonocytic cells. J. Exp. Med. 186:1809-1818.

27. Levitsky, V., de Campos-Lima, P.O., Frisan, T., and Masucci, M.G. 1998 The clonal composition of a peptide-specific oligoclonal CTL repertoire selected in response to persistent EBV infection is stable over time. J. Immunol. 161:594-601.

28. Heid, C.A., Stevens, J., Livak, K.J., and Williams, P.M. 1996. Real time quantitative PCR. Genome Res. 6:986-994.

29. Fink, L., et al. 1998. Real-time quantitative RT-PCR after laser-assisted cell picking. Nat. Med. 4:1329-1333.

30. Sivori, S., et al. 1996. CD94 functions as a natural killer cell inhibitory receptor for different HLA class I alleles: identification of the inhibitory form of CD94 by the use of novel monoclonal antibodies. Eur. J. Immunol. 26:2487-2492.

31. Gronberg, A., Ferm, M., Tsai, L., and Kiessling, R. 1989. Interferon is able to reduce tumor cell susceptibility to human lymphokine-activated killer (LAK) cells. Cell. Immunol. 118:10-21.

32. Mingari, M.C., et al. 1995. Cytolytic T lymphocytes displaying natural killer (NK)-like activity: expression of NK-related functional receptors for HLA class I molecules (p58 and CD94) and inhibitory effect on the TCR-mediated target cell lysis or lymphokine production. Int. Immunol. 7:697-703.

33. Llano, M., et al. 1998. HLA-E-bound peptides influence recognition by inhibitory and triggering CD94/NKG2 receptors: preferential response to an HLA-G-derived nonamer. Eur. J. Immunol. 28:2854-2863.

34. Lee, N., et al. 1995. The membrane-bound and soluble forms of HLA-G bind identical sets of endogenous peptides but differ with respect to TAP association. Immunity. 3:591-600.

35. Speiser, D.E., et al. 1999. CD28-negative cytolytic effector T cells frequently express NK receptors and are present at variable proportions in circulating lymphocytes from healthy donors and melanoma patients. Eur. J. Immunol. 29:1990-1999.

36. Mingari, M.C., et al. 1998. HLA class I-specific inhibitory receptors in human $\mathrm{T}$ lymphocytes: interleukin 15-induced expression of CD94/NKG2A in superantigen- or alloantigen-activated CD8+ T cells. Proc. Natl. Acad. Sci. USA. 95:1172-1177.

37. Bertone, S., et al. 1999. Transforming growth factor-beta-induced expression of CD94/NKG2A inhibitory receptors in human $\mathrm{T}$ lymphocytes. Eur. J. Immunol. 29:23-29.

38. Sallusto, F., Lenig, D., Forster, R., Lipp, M., and Lanzavecchia, A. 1999. Two subsets of memory $\mathrm{T}$ lymphocytes with distinct homing potentials and effector functions. Nature. 401:708-712.

39. Le Drean, E., et al. 1998. Inhibition of antigen-induced T cell response and antibody-induced NK cell cytotoxicity by NKG2A: association of NKG2A with SHP-1 and SHP-2 protein-tyrosine phosphatases. Eur. J. Immunol. 28:264-276.

40. Mingari, M.C., Moretta, A., and Moretta, L. 1998. Regulation of KIR expression in human $\mathrm{T}$ cells: a safety mechanism that may impair protective T-cell responses. Immunol. Today 19:153-157.

41. Young, N.T., Uhrberg, M., Phillips, J.H., Lanier, L.L., and Parham, P. 2001. Differential expression of leukocyte receptor complex-encoded Ig-like receptors correlates with the transition from effector to memory CTL. J. Immunol. 166:3933-3941.

42. Vely, F., et al. 2001. Regulation of inhibitory and activating killer-cell Iglike receptor expression occurs in T cells after termination of TCR rearrangements. J. Immunol. 166:2487-2494.

43. Gustafson, K.S., and Ginder, G.D. 1996. Interferon-gamma induction of the human leukocyte antigen-E gene is mediated through binding of a complex containing STAT1alpha to a distinct interferon-gammaresponsive element. J. Biol. Chem. 271:20035-20046.

44. Cerboni, C., et al. 2001. Synergistic effect of IFN-gamma and human cytomegalovirus protein UL40 in the HLA-E-dependent protection from NK cell-mediated cytotoxicity. Eur. J. Immunol. 31:2926-2935.

45. Vales-Gomez, M., Reyburn, H.T., Erskine, R.A., Lopez-Botet, M., and Strominger, J.L. 1999. Kinetics and peptide dependency of the binding of the inhibitory NK receptor CD94/NKG2-A and the activating receptor CD94/NKG2-C to HLA-E. EMBO J. 18:4250-4260.

46. Navarro, F., et al. 1999. The ILT2(LIR1) and CD94/NKG2A NK cell receptors respectively recognize HLA-G1 and HLA-E molecules coexpressed on target cells. Eur. J. Immunol. 29:277-283.

47. Real, L.M., et al. 1999. Expression of HLA G in human tumors is not a frequent event. Int. J. Cancer. 81:512-518.

48. Marincola, F.M., Jaffee, E.M., Hicklin, D.J., and Ferrone, S. 2000. Escape of human solid tumors from T-cell recognition: molecular mechanisms and functional significance. Adv. Immunol. 74:181-273.

49. Freedman, R.S., et al. 2000. Clinical and biological effects of intraperitoneal injections of recombinant interferon-gamma and recombinant interleukin 2 with or without tumor-infiltrating lymphocytes in patients with ovarian or peritoneal carcinoma. Clin. Cancer Res. 6:2268-2278.

50. Meyskens, F.L., Jr., et al. 1995. Randomized trial of adjuvant human interferon gamma versus observation in high-risk cutaneous melanoma: a Southwest Oncology Group study. J. Natl. Cancer Inst. 87:1710-1713.

51. Pisa, P., et al. 1992. Selective expression of interleukin 10, interferon gamma, and granulocyte-macrophage colony-stimulating factor in ovarian cancer biopsies. Proc. Natl. Acad. Sci. USA. 89:7708-7712

52. Koh, C.Y., et al. 2001. Augmentation of antitumor effects by NK cell inhibitory receptor blockade in vitro and in vivo. Blood. 97:3132-3137. 